\title{
Complications after bronchoscopic lung volume reduction
}

\author{
Daniel Franzen $^{1}$, Gilles Straub ${ }^{1}$, Lutz Freitag $^{2}$ \\ ${ }^{1}$ Interventional Lung Center, Department of Pulmonology, University Hospital Zurich, Zurich, Switzerland; ${ }^{2}$ Clinic St. Anna, Hirslanden Group, \\ Lucerne, Switzerland \\ Contributions: (I) Conception and design: D Franzen, L Freitag; (II) Administrative support: G Straub; (III) Provision of study materials or patients: \\ G Straub, D Franzen; (IV) Collection and assembly of data: G Straub, D Franzen; (V) Data analysis and interpretation: All authors; (VI) Manuscript \\ writing: All authors; (VII) Final approval of manuscript: All authors. \\ Correspondence to: Dr. Daniel Franzen, MD. Department of Pulmonology, University Hospital Zurich, Raemistrasse 100, 8091 Zurich, Switzerland. \\ Email: daniel.franzen@usz.ch.
}

\begin{abstract}
Bronchoscopic lung volume reduction (BLVR) has been demonstrated an efficient and safe alternative to surgery in multiple randomized trials and retrospective cohort studies. However, despite its minimal invasiveness BLVR is not without potential harm. Complications and their incidents differ significantly between the individual BLVR techniques (valves, coils, vapour or sealant) which are bearing varying device- and intervention-dependent risks. Interventional pulmonologists must be aware of potential side effects and their management to anticipate and ensure highest quality treatment of the severely ill emphysema patient.
\end{abstract}

Keywords: Emphysema; chronic obstructive pulmonary disease (COPD); lung volume reduction; bronchoscopy; complications

Submitted Apr 23, 2018. Accepted for publication Jun 11, 2018.

doi: $10.21037 /$ jtd.2018.06.66

View this article at: http://dx.doi.org/10.21037/jtd.2018.06.66

\section{Introduction}

Lung volume reduction surgery (LVRS) has been shown to improve pulmonary function, maximal workload, quality of life, and even survival in patients with both upperlobe predominant emphysema and low base-line exercise capacity (1). However, among patients with non-upper-lobe emphysema distribution, mortality was higher in the LVRS group compared to the best supportive care group due to several reasons (1). This finding, in addition to a publication from the same authors titled "Patients at high risk of death after lung-volume-reduction surgery (2)" have led to a global decline of LVRS procedures (3). Simultaneously, new non-invasive approaches with bronchoscopic techniques for lung volume reduction have been emerging because lower mortality and complication rates seemed reasonable $(4,5)$. Since then, essentially four methods of bronchoscopic lung volume reduction (BLVR) have been investigated in multiple, randomized trials and cohort studies. Plugs and valves are used to create lobar atelectases, foam or vapor is applied in order to shrink overinflated segments and coils are placed aiming to increase the elastic recoil of the emphysematous lung parenchyma. Details of these techniques concerning indications, patient selection and efficacy are described elsewhere in this issue. In the following sections, we discuss relevant device- and procedure-related complications and their management.

\section{Complications after valve treatment}

The primary goal of BLVR using endo- or intrabronchial valves is to close the feeding bronchi of an overinflated lobe, thereby achieving lobar atelectasis, which eventually leads to the desired lung volume reduction effect. Device- and procedure-related complications are listed in Table 1.

The most prevalent complication is pneumothorax in up to $26 \%$ of cases due to compensatory over-expansion of the ipsilateral untreated lobe (6). However, the risk of a lifethreatening tension pneumothorax is roughly unknown. In fact, it remains debatable, if a pneumothorax after valve 
Table 1 Device- and procedure-related complications after endo-/ intrabronchial valve placement

\begin{tabular}{lc}
\hline Complication & Rates $(\%)$ \\
\hline Pneumothorax $^{*}$ & $8-26$ \\
COPD exacerbation & $5-64$ \\
Pneumonia & $0-9$ \\
Hemoptysis & 2 \\
Valve migration & $5-6$ \\
Valve expectoration & $0-16$ \\
Granulation tissue formation & 3 \\
Death & $0-3$ \\
\hline
\end{tabular}

Reported complication rates according to VENT, BeLieVeRHIFi, STELVIO, IMPACT and TRANSFORM (6-11). *, P<0.0001 compared to control group; not significant compared to control group. COPD, chronic obstructive pulmonary disease.

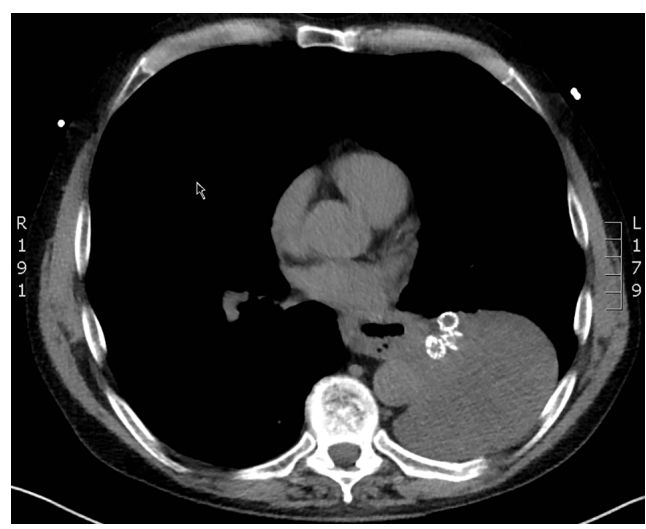

Figure 1 Post-valvular pneumonia. Axial chest CT (mediastinal window) with evidence of severe consolidation 6 months after successful BLVR with endobronchial valves in the left lower lobe in a 51-year-old patient with $\alpha 1$-antitrypsin deficiency and severe emphysema, suggesting "post-valvular" pneumonia. After valve removal and antibiotic treatment, the patient fully recovered. Several months later, successful lung volume reduction surgery was performed. BLVR, bronchoscopic lung volume reduction.

treatment should be regarded as a complication or could be called a treatment effect, analogously to chest tube drainage after LVRS. However, there are several publications reporting strategies to anticipate, predict or treat pneumothoraces after valve treatment. In one prospective study, Herzog et al. investigated the effect of modified medical care on pneumothorax rate compared to standard care. Their approach includes bedrest for 48 hours after valve placement and cough suppressant therapy (12). They found that modified medical care could significantly lower the pneumothorax rate from $25 \%$ to $5 \%$ (12). In addition, Gompelmann et al. identified low attenuation volume of the ipsilateral untreated lobe, ipsilateral untreated lobe volume/hemithorax volume ratio, emphysema type, pleural adhesions and residual volume as significant predictors of pneumothorax (13). However, no single risk factor has been identified that could influence the decision making whether and how a patient should be treated or excluded from valve therapy because of a potentially fatal tension pneumothorax. Therefore, it has become common practice to not treat these patients as outpatients but keep them in the hospital under surveillance having an emergency drainage set available. Finally, an expert panel has recently published recommendations for the treatment of a pneumothorax after valve treatment (14).

Interestingly, according to the major randomized trials for valve treatment (VENT, BeLieVeR-HIFi, STELVIO, IMPACT and TRANSFORM), other serious adverse events, including chronic obstructive pulmonary disease (COPD) exacerbation, pneumonia, hemoptysis and death did not show significantly higher prevalences in valve treated patients compared to controls (6-10). However, care providers must be aware of potentially fatal complications, such as post-valvular pneumonia with septic shock (Figure 1) or severe hemoptysis with subsequent respiratory failure (Figure 2). Recently, a case of severe hypersensitivity pneumonitis in a patient with Nickel allergy treated with endobronchial valves (15). In addition, the authors detected loss of Nickel ions from valves in in vitro experiments. Although a causative relationship remains indeed speculative and valves are merely made of Nitinol (Nickel alloy) rather than Nickel, valve placement in patients with distinct symptoms of Nickel allergy should only be done with caution.

According to the 1-year follow-up data from the STELVIO trial, valve replacement or permanent valve removal were necessary in $17 \%$ each for different reasons (valve expectoration, valve migration, pneumonia, increased complaints without benefit, recurrent pneumothorax, torsion of bronchus, and granulation tissue formation) (11). The latter is to date an unsolved problem, which is also frequently seen after airway stenting (Figure 3). The interaction between the mucosa and any foreign body bears the risk of adverse tissue reactions which in case of valves can lead to impaired functions. Companies are currently working on drug-eluting stents to prevent overwhelming 


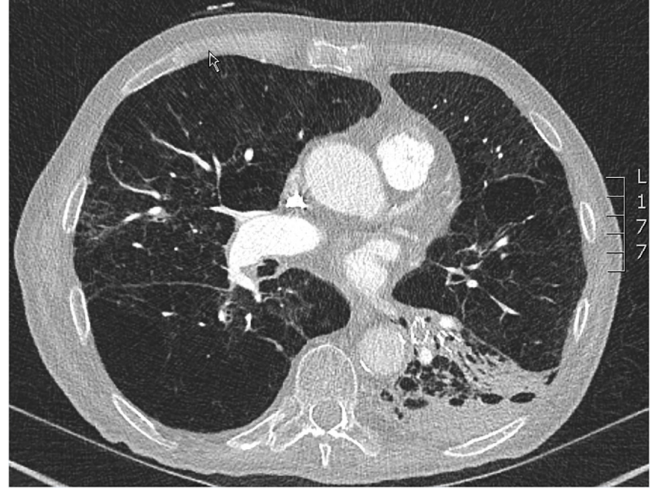

Figure 2 Bleeding after BLVR with valves. Axial chest CT (lung window) with consolidation and ground-glass opacities in a 73-year-old patient with severe emphysema presenting with major hemoptysis 3 years after successful BLVR with endobronchial valves in the left lower lobe. At emergency bronchoscopy, the cause of bleeding was mucosal erosion. Therefore, the valves had to be removed and the bleeding was stopped with argon plasma coagulation after topical instillation of a vasoconstrictor. BLVR, bronchoscopic lung volume reduction.

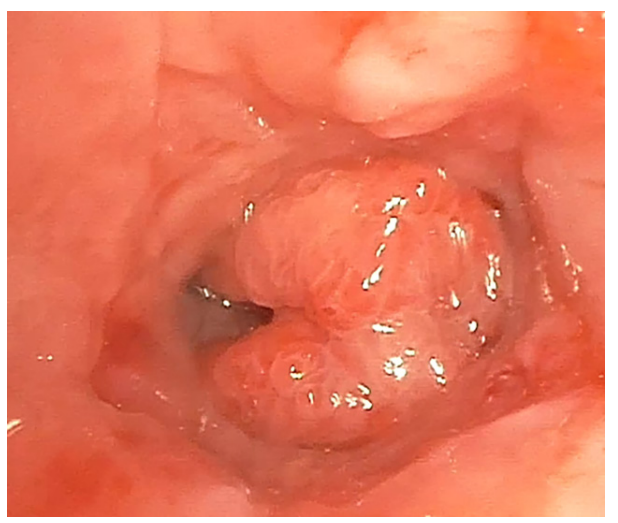

Figure 3 Granulation tissue. Bronchoscopic view into anterior segment of left lower lobe 9 months after unsuccessful BLVR with endobronchial valves in the left lower lobe in a 72-year-old patient with evidence of granulation tissue formation in front of endobronchial valve (valve covered by granulation tissue). BLVR, bronchoscopic lung volume reduction.

granulation tissue formation, which may also be translated into valve technology.

\section{Complications after coil treatment}

The most frequent complication after coil implantation is

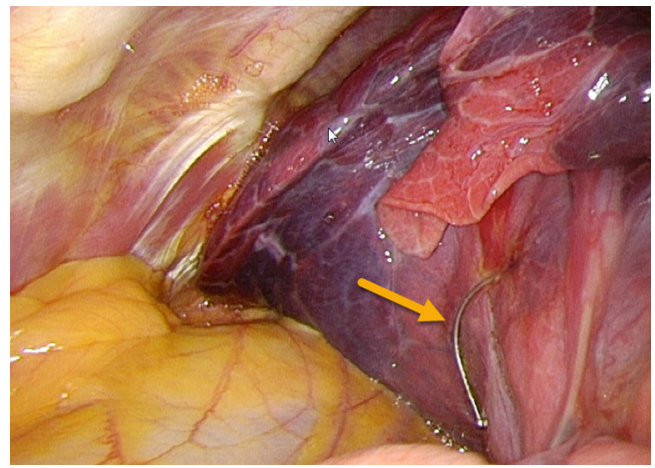

Figure 4 Coil perforation (arrow). Thoracoscopic view on left upper lobe/lingual with perforated lung volume reduction coils 2 days after BLVR. The coils could be easily removed, and the perforated area was sealed with wedge resection. BLVR, bronchoscopic lung volume reduction.

COPD exacerbation in up to $18 \%$ of cases (16). However, COPD exacerbations were not significantly more prevalent compared to usual care in either of the three major randomized trials (16-18). By contrast, pneumonia or lower tract respiratory infections were significantly more prevalent in coil treated patients (up to $20 \%$ ) compared to usual care $(4.5 \%)(16,17)$. A new entity has emerged that runs any the term: "coil-associated opacities". The radiological finding is difficult to distinguish from pneumonia. The awareness has been increasing after the publications of these trials, the clinical importance is not really clear. In fact, the true pathogenesis of these opacities is unknown. In addition, it is a matter of debate, if coil-associated opacities may enhance the beneficial effect on pulmonary function on the long-term. The incidences of pneumothoraces after coil-implantation range between 5-9\% (16-18). In rare cases, pneumothorax can be caused be direct coil perforation of lung structures, even spiking into the pleural space (Figure 4). In two of our cases thoracoscopic coil removal and wedge resection were necessary to treat these iatrogenic pneumothoraces. It is debatable whether we had placed the coils to far distally in first place but other clinicians should be aware of these possible complications requiring the help of thoracic surgeons. Hemoptysis after coil implantation is reported in up to $3 \%$ of cases (17). The majority of hemoptysis cases are of light to moderate degree with spontaneous termination of the bleeding. Only in very rare cases, bronchoscopic intervention with topic instillation of vasoconstrictors or insertion of a bronchus blocker 
is needed. In a single case refractory we had to perform selective bronchial artery embolization to stop the bleeding.

\section{Complications after thermic and chemical lung volume reduction}

Details of chemical LVR using a lung sealant (AeriSeal ${ }^{\circledR}$, PulmonX, Redwood, CA, USA) and bronchoscopic thermal vapour ablation (BTVA, Bronchus Inc.) are both explained elsewhere in this issue. In an early safety study on 20 patients, there was a $5 \%$ procedure-related mortality after chemical LVR due to tension pneumothorax and subsequent nosocomial sepsis (19). COPD exacerbations and lower tract respiratory tract infections or pneumonitis were seen in $15 \%$ each. Another small study demonstrated no treatmentrelated deaths in a series of 25 patients using endoscopic lung sealant (20) but COPD exacerbations occurred in $40 \%$ of these patients. Apart from that, the authors were to first to show that sealant therapy was routinely associated with a flu-like symptoms beginning 8-24 hours following treatment with elevated inflammation markers and dyspnea in all investigated patients. These signs usually disappeared after 24 hours or were successfully treated with antipyretics and corticosteroids if they lasted more than 24 hours (20). Currently, endoscopic lung sealant treatment is not commercially available. Studies on a safer application with altered ingredient dosages are under way.

Like foam sealant treatment vapor application is another irreversible measure that causes volume reduction by a combination of intended obstruction and induction of scarring. After BTVA, the most frequent complications were lower respiratory tract infection or pneumonia in $18-25 \%$, followed by COPD exacerbation in $20-24 \%$ and self-limiting hemoptysis in 2-7\% $(21,22)$. Most of the respiratory adverse events occurred in the first 90 days after treatment, and all of them were treated with standard medical care with corticosteroids and antibiotics. Only a minority of patients $(7 \%)$ in the treatment arm required significant medication intervention (intensive care unit stay or re-bronchoscopy) (23).

\section{Conclusions}

Despite its minimal invasiveness and using a natural orifice, BLVR is not without a considerable risk of harm. However, with proper patient selection and education, continuing training of treating physicians and allied health care professionals with in-depth knowledge on possible complications, and ongoing clinical studies and prospective registers, non-invasive bronchoscopic treatments can be offered to a wide range of patients with emphysema with an acceptable safety profile.

Additional comment: judging risks and severity of side effects depends on the perspective. The authors' experience is that emphysema who really suffer from their terrible disease are in most cases prepared to accept the risks of possible complications even if those are fully explained to them as they do not want to continue living with their disabilities.

\section{Acknowledgements}

None.

\section{Footnote}

Conflicts of Interest: The authors have no conflicts of interest to declare.

\section{References}

1. Fishman A, Martinez F, Naunheim K, et al. A randomized trial comparing lung-volume-reduction surgery with medical therapy for severe emphysema. $\mathrm{N}$ Engl J Med 2003;348:2059-73.

2. Fishman A, Fessler H, Martinez F, et al. Patients at high risk of death after lung-volume-reduction surgery. $\mathrm{N} \mathrm{Engl}$ J Med 2001;345:1075-83.

3. Criner GJ, Cordova F, Sternberg AL, Martinez FJ. The National Emphysema Treatment Trial (NETT) Part II: Lessons learned about lung volume reduction surgery. Am J Respir Crit Care Med 2011;184:881-93.

4. Ingenito EP, Reilly JJ, Mentzer SJ, et al. Bronchoscopic volume reduction: a safe and effective alternative to surgical therapy for emphysema. Am J Respir Crit Care Med 2001;164:295-301.

5. Toma TP. The flexible bronchoscopic approach to lung volume reduction. Pneumologia 2001;50:97-100.

6. Valipour A, Slebos DJ, Herth F, et al. Endobronchial Valve Therapy in Patients with Homogeneous Emphysema. Results from the IMPACT Study. Am J Respir Crit Care Med 2016;194:1073-82.

7. Herth FJ, Noppen M, Valipour A, et al. Efficacy predictors of lung volume reduction with Zephyr valves in a European cohort. Eur Respir J 2012;39:1334-42.

8. Davey C, Zoumot Z, Jordan S, et al. Bronchoscopic lung 
volume reduction with endobronchial valves for patients with heterogeneous emphysema and intact interlobar fissures (the BeLieVeR-HIFi study): a randomised controlled trial. Lancet 2015;386:1066-73.

9. Klooster K, Ten Hacken NH, Hartman JE, et al. Endobronchial Valves for Emphysema without Interlobar Collateral Ventilation. N Engl J Med 2015;373:2325-35.

10. Kemp SV, Slebos DJ, Kirk A, et al. A Multicenter Randomized Controlled Trial of Zephyr Endobronchial Valve Treatment in Heterogeneous Emphysema (TRANSFORM). Am J Respir Crit Care Med 2017;196:1535-43.

11. Klooster K, Hartman JE, Ten Hacken NH, et al. OneYear Follow-Up after Endobronchial Valve Treatment in Patients with Emphysema without Collateral Ventilation Treated in the STELVIO Trial. Respiration 2017;93:112-21.

12. Herzog D, Poellinger A, Doellinger F, et al. Modifying Post-Operative Medical Care after EBV Implant May Reduce Pneumothorax Incidence. PLoS One 2015;10:e0128097.

13. Gompelmann D, Lim HJ, Eberhardt R, et al. Predictors of pneumothorax following endoscopic valve therapy in patients with severe emphysema. Int J Chron Obstruct Pulmon Dis 2016;11:1767-73.

14. Valipour A, Slebos DJ, de Oliveira HG, et al. Expert statement: pneumothorax associated with endoscopic valve therapy for emphysema--potential mechanisms, treatment algorithm, and case examples. Respiration 2014;87:513-21.

15. Franzen DP, Lang C, Agorastos N, et al. Evaluation of Nickel Release from Endobronchial Valves as a Possible Cause of Hypersensitivity Pneumonitis in a Patient Treated with Bronchoscopic Lung Volume Reduction. Int

Cite this article as: Franzen D, Straub G, Freitag L. Complications after bronchoscopic lung volume reduction. J Thorac Dis 2018;10(Suppl 23):S2811-S2815. doi: 10.21037/ jtd.2018.06.66
Arch Allergy Immunol 2017;174:144-50.

16. Deslée G, Mal H, Dutau H, et al. Lung Volume Reduction Coil Treatment vs Usual Care in Patients With Severe Emphysema: The REVOLENS Randomized Clinical Trial. JAMA 2016;315:175-84.

17. Sciurba FC, Criner GJ, Strange C, et al. Effect of Endobronchial Coils vs Usual Care on Exercise Tolerance in Patients With Severe Emphysema: The RENEW Randomized Clinical Trial. JAMA 2016;315:2178-89.

18. Shah PL, Zoumot Z, Singh S, et al. Endobronchial coils for the treatment of severe emphysema with hyperinflation (RESET): a randomised controlled trial. Lancet Respir Med 2013;1:233-40.

19. Kramer MR, Refaely Y, Maimon N, et al. Bilateral endoscopic sealant lung volume reduction therapy for advanced emphysema. Chest 2012;142:1111-7.

20. Herth FJ, Eberhardt R, Ingenito EP, Gompelmann D. Assessment of a novel lung sealant for performing endoscopic volume reduction therapy in patients with advanced emphysema. Expert Rev Med Devices 2011;8:307-12.

21. Snell G, Herth FJ, Hopkins P, et al. Bronchoscopic thermal vapour ablation therapy in the management of heterogeneous emphysema. Eur Respir J 2012;39:1326-33.

22. Herth FJ, Valipour A, Shah PL, et al. Segmental volume reduction using thermal vapour ablation in patients with severe emphysema: 6-month results of the multicentre, parallel-group, open-label, randomised controlled STEPUP trial. Lancet Respir Med 2016;4:185-93.

23. Shah PL, Gompelmann D, Valipour A, et al. Thermal vapour ablation to reduce segmental volume in patients with severe emphysema: STEP-UP 12 month results. Lancet Respir Med 2016;4:e44-e45. 